\title{
99mTc EDHP bone scintiscans in healthy young adults and patients with Reiter's syndrome
}

\author{
KLAUS K. BANDILLA \\ From the Deutsche Klinik für Diagnostik, Wiesbaden, West Germany
}

In agreement with Professor Russell we find increased radioisotope activity over the sacroiliac joints when scanning patients with Reiter's syndrome (RS) with ${ }^{99 m}$ Technetium stannous pyrophosphate. We use a similar quantitative technique calculating the sacroiliac uptake ratio. Increased isotope uptake was also found on clinically non-involved sacroiliac joints. Indeed, the value of the scan is somewhat questionable in patients under 30 years of age, since young patients tend to have high sacroiliac ratios (that is, of 1.60 and over) with or without any clinical signs of disease (Figure).

The Figure shows an attempt to correlate the sacroiliac uptake ratio with the age of the patient. The black part of the column represents patients with ankylosing spondylitis or one of the 'rheumatoid variants' with potential sacroiliac joint involvement. More than $50 \%$ of those aged under 30 have a high ratio even though two-thirds had no clinical or radiological evidence of sacroiliac joint disease. In those aged 30 to 50 we hardly ever find a high sacroiliac ratio. To find, without discrimination, a high uptake ratio in many patients aged under 30 is especially perturbing, since this is a group where the early diagnosis of ankylosing spondylitis is very difficult and where any additional diagnostic help would be of particular value. Yet, in our experience, the sacroiliac joint scan seems to be of only limited use in this young age group.

Typing for HLA-B27 seems to have more diagnostic significance, especially in patients aged under 30. Among 50 patients positive for B27 22 (44\%) had a low uptake ratio. Among these 22 were five with clinically active disease. In nine $(18 \%)$ the scan was indifferent, and in $19(38 \%)$ the sacroiliac ratio

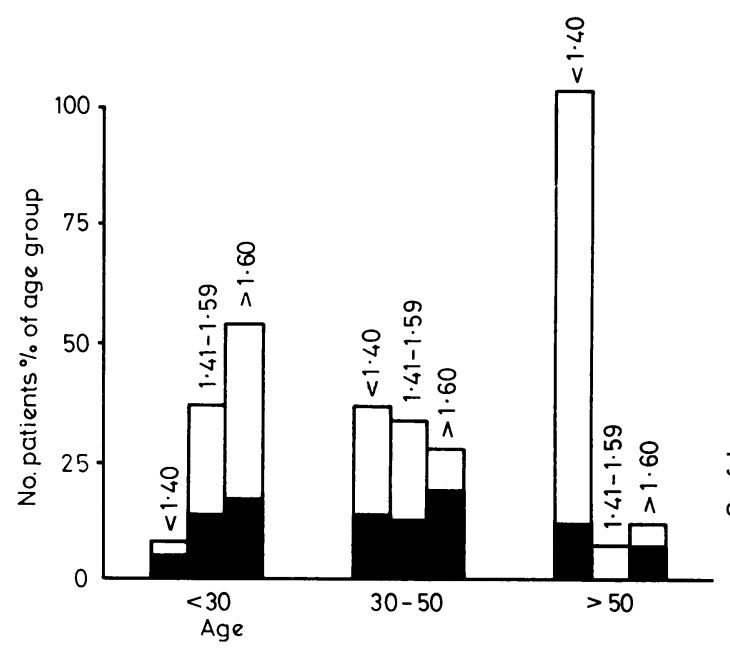

Figure ${ }^{99 m} T c$ scintiscan in healthy young adults and patients with RS: Pathological sacroiliac ratio of $>1.60$ in healthy controls (white) versus patients with ankylosing spondylitis or rheumatoid variants (black). Note significant proportion of 'false positives' in healthy controls aged under 30 .

was high. Only 10 of the latter had clinical and/or radiographic evidence of sacroiliitis. Moreover, a pathological sacroiliac ratio of over 1.60 was found in five out of 37 patients with degenerative joint disease or chronic non-inflammatory back pain.

Thus, we were unable to detect among our patients any characteristic pattern of bone scanning which could be helpful in diagnosing active sacroiliitis, early ankylosing spondylitis, or RS. 